\section{MR Imaging of the Ankle and Hindfoot}

MR imaging is the diagnostic modality of choice for the evaluation of traumatic ligamentous and tendinous injuries of the ankle and hindfoot. MR imaging is also valuable in the detection of occult bony trauma, osteochondral injuries, avascular necrosis, osteomyelitis, and a variety of other osseous conditions. Exquisite contrast resolution, noninvasiveness, and multiplanar capabilities are unique features that make MR imaging a powerful diagnostic technique. The authors employ the Basic Protocol using $T_{1^{-}}$ and $T_{2}$-weighted sequences and short tau inversion recovery (STIR) imaging. Additional imaging following intravenous gadolinium is indicated in selected cases.

If gadolinium contrast is used, whether intravenous or intra-articular, pre- and postcontrast $T_{1}$-weighted sequences in each appropriate plane will be needed for optimal evaluation/comparison.

\section{MR EXAMINATION OF THE ANKLE AND HINDFOOT}

Most reports on MR imaging of the ankle and foot available in the literature are based on studies performed on high-strength (1.5 T) magnets (Kneeland, 1994; Zlatkin, 1999). The following sequences described herein are based on the author's experience with a GE 1.5 T scanner, but are expected to be applicable to other equipment manufacturers. The entire protocol takes $\sim 21 \mathrm{~min}$ to perform. If post-gadolinium imaging is obtained, 8 additional min are required.

Table A25.1.1 lists the six sequences necessary to perform the Basic Protocol, along with one optional sequence. Stepwise instructions for performing the imaging protocol are provided. This protocol should be easily acquired on most clinical systems.

Table A25.1.2 lists the hardware necessary to perform the procedure, along with the appropriate parameters. The available gradient strength will depend on the scanner, and the echo times given below may be varied accordingly (the smaller gradient strength, the longer the echo time for a particular scan).

When using mid/low field scanners, adjustments to the imaging protocols need to be made in order to compensate for the lower signal-to-noise ratio (SNR) related to lower-field strength and gradient strength. In general, MR examinations obtained in mid/low field machines require longer acquisition times and a larger field of view. Signal abnormalities related to increased water content in tissue must be evaluated using STIR sequences since frequency-selective fat saturation cannot be obtained when using these systems.

NOTE: Be sure that technologists and nurses have immediate access to any emergency equipment that may be relevant to a given study, or that may be needed for a particular patient, such as crash carts or oxygen.

\section{Materials}

Extravascular contrast agent (e.g., Omniscan, Magnevist, Prohance)

Normal saline $(0.9 \% \mathrm{NaCl})$, sterile

\section{Set up patient and equipment}

1. Interview the patient to assess for contraindications such as cardiac pacemakers or other implants containing ferromagnetic materials. Also, determine if the patient will need sedation medication requiring the use of monitoring equipment.
UNIT A25.1

BASIC

PROTOCOL
Ankle and Foot

A25.1.1

Supplement 15 
Type of weighting and sequences

1. Gradient echo three-plane positioning scout

2. $T_{1}$-weighted spin echo

3. $T_{2}$-weighted fast spin echo (FSE)

4. $T_{1}$-weighted spin echo

5. Fat-suppressed proton density weighted fast spin echo

6. Turbo STIR

Optional sequence

7. Post-gadolinium fat suppressed $T_{1}$-weighted spin echo
Imaging plane

Sagittal, coronal, and transverse

Transverse

Transverse

Coronal

Coronal

Sagittal

Table A25.1.2 Equipment Parameters

\begin{tabular}{ll}
\hline Coil type & Quadrature extremity coil \\
Gradient coil strength & $25 \mathrm{mT} / \mathrm{m}$ (or whatever the system permits) \\
Cardiac gating & No \\
Respiratory gating & No \\
Peripheral monitoring & For safety only \\
Respirator/oxygen & If required by the patient \\
Motion cushions & Useful \\
\hline
\end{tabular}

A screening form should be filled and signed by each patient or legal guardian prior to scanning in a magnetic resonance system. Generally, standard screening forms (APPENDIX 1) are used for all patients scanned in a magnetic resonance system.

The presence of ferromagnetic material in a patient may represent a health hazard to the patient while he or she is inside the magnet and/or deteriorate image quality. The safety of certain ferromagnetic materials can be found in Shellock and Kanal (1996).

Patients with previous metal exposure to the eyes should have radiographs of the orbits in order to exclude metallic foreign bodies that have not been removed prior to placing them in the magnetic field.

A friend or family member, who can sit in the room during the scan and comfort the patient as needed, may accompany patients into the magnet room. This companion must be screened as well to ensure the absence of loose metal objects on the body or clothing.

2. If the procedure is a research protocol, have the patient sign any necessary consent forms.

3. Have the patient remove jewelry and change into a gown to eliminate any metal that might be found in clothing.

All personal belongings should be secured properly during the examination.

4. Have the patient wash off any mascara and other makeup to avoid local tissue heating and image artifacts.

5. Inform the patient about what will occur during the procedure, what he or she will experience while in the magnet, and how to behave.

a. If earphones or headphones are used to protect the ears from the loud sounds produced by the gradients, the patient will be asked to wear these, but will be able to communicate with you at any time during the imaging.

MR Imaging of the Ankle and Hindfoot

A25.1.2

b. The patient will be given a safety squeeze-bulb or similar equipment to request assistance at any time (demonstrate how this works). 
c. For good results, the patient should not talk, and should avoid or minimize other movement, during each scan-i.e., as long as the banging sounds continue. Between scans, talking is allowed in most cases, but should be avoided when comparative positional studies are being performed; the patient will be informed when this is the case.

d. Nevertheless, the patient may call out at any time if he or she feels it necessary.

6. Ask the patient to remain perfectly still during imaging acquisition and also explain to him or her not to move between imaging sequences.

7. Have the patient lie on the table, with the feet toward the bore of the magnet. For imaging of the ankle and hindfoot, the authors recommend to lie the patient supine with the ankle in neutral position. Either before or right after the patient lies down, set up any triggering devices or other monitoring equipment that is to be used.

The ankle in neutral position has an inherent $10^{\circ}$ to $20^{\circ}$ of plantar flexion and $10^{\circ}$ to $30^{\circ}$ of external rotation. This mild degree of plantar flexion is useful for eliminating the magic angle effect when examining the flexor and extensor tendons of the ankle and hindfoot. Increasing the thickness of the fat planes between the peroneus brevis and longus tendons, and displacing the flexor and peroneal tendons away from the bones, are other advantages of imaging the ankle in plantar flexion.

Mechanical stabilization of the ankle is paramount for good image quality. Immobilization should be achieved by using foam sponges between the ankle and the coil and Velcro straps on the leg outside the coil.

8. Center the ankle to be examined in the extremity coil using the infrared light pointing to the ankle joint level.

9. Advance the patient table to isocenter.

Once this step has been performed, so long as the patient does not move on the table, the table itself can be moved and then placed in the same position as before without jeopardizing the positioning of one scan relative to another.

10. If the patient is unable to hold still, provide an appropriate sedative if indicated.

Table A25.1.3 Parameters for Scout Scan

\begin{tabular}{ll}
\hline Patient position & $\begin{array}{l}\text { Supine } \\
\text { Gradient echo }\end{array}$ \\
Scan type & Three planes \\
Imaging plane (orientation) & Magnet isocenter \\
Central slice or volume center & $4.2 \mathrm{msec}$ \\
Echo time $\left(T_{\mathrm{E}}\right)$ & $12.1 \mathrm{msec}$ \\
Repeat time $\left(T_{\mathrm{R}}\right)$ & $30^{\circ}$ \\
Flip angle $(\mathrm{FA})$ & $240 \mathrm{~mm}, 240 \mathrm{~mm}$ \\
Fields of view $\left(\mathrm{FOV}_{x}, \mathrm{FOV}_{y}\right)$ & $1.88 \mathrm{~mm}, 1.88 \mathrm{~mm}$ \\
Resolution $(\Delta x, \Delta y)$ & 128,128 \\
Number of data points collected $\left(N_{x}, N_{y}\right)$ & $4 \mathrm{~mm}$ \\
Slice thickness $(\Delta z)$ & $9(3 \mathrm{in}$ each of $3 \mathrm{cardinal}$ planes $)$ \\
Number of slices & $1 \mathrm{~mm}$ \\
Slice gap & 1 \\
Number of acquisitions $\left(N_{\text {acq }}\right)$ & No \\
Swap read and phase encoding & Not applicable \\
Slice location & Not applicable \\
Saturation pulses & $18 \mathrm{sec}$ \\
Scan time &
\end{tabular}




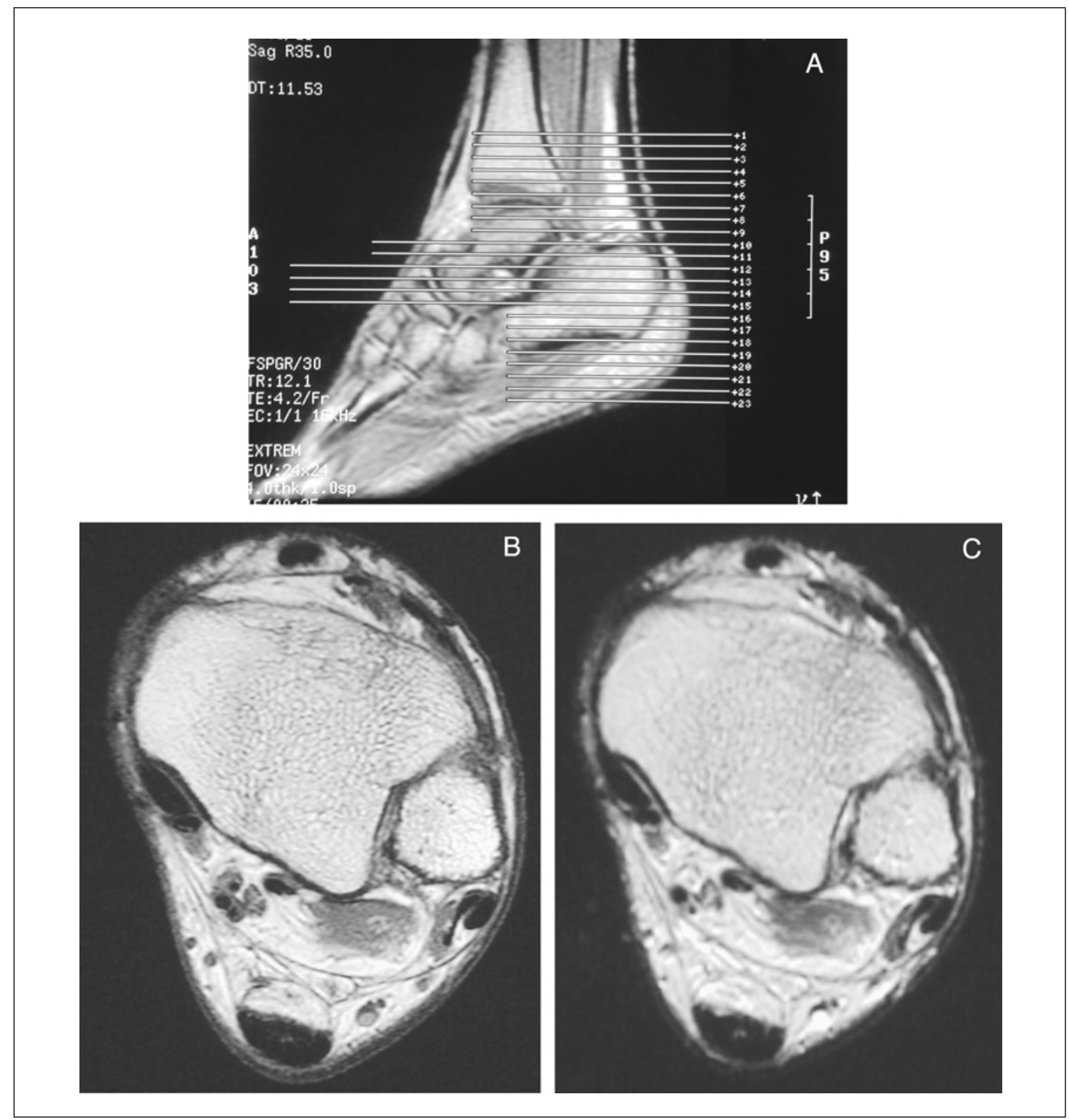

Figure A25.1.1 Transverse imaging plane. (A) Using the sagittal localizer, transverse $T_{1}$-weighted spin echo images are prescribed covering the Achilles tendon and entire calcaneus. The ankle is in neutral position with $20^{\circ}$ of plantar flexion. (B) Transverse $T_{1}$-weighted image through the distal tibial plafond. Note exquisite anatomic detail. (C) Transverse $T_{2}$-weighted FSE image at the same level as $\mathbf{B}$.

MR Imaging of the Ankle and Hindfoot

\section{Sequence 1: Three-plane positioning scout}

11. To verify the patient's position, run the scout scan to ensure correct location of the ankle in 3-D using the imaging parameters provided in Table A25.1.3.

This sequence will be used to set up coverage of the area of interest. Most MR machines can be programmed to acquire the scout images automatically after coil tuning or after the patient has been placed in the isocenter.

\section{Sequence 2: Transverse $T_{1}$-weighted spin echo}

12. Using the images generated in sequence 1, prescribe transverse images to provide complete coverage of the ankle and hindfoot (Fig. A25.1.1). Run sequence 2 according to Table A25.1.4. 
Table A25.1.4 Parameters for Transverse $T_{1}$-Weighted Spin Echo

Patient position

Scan type

Imaging plane (orientation)

Central slice or volume center

Echo time $\left(T_{\mathrm{E}}\right)$

Repeat time $\left(T_{\mathrm{R}}\right)$

Flip angle (FA)

Fields of view $\left(\mathrm{FOV}_{x}, \mathrm{FOV}_{y}\right)$

Resolution $(\Delta x, \Delta y)$

Number of data points collected $\left(N_{x}, N_{y}\right)$

Slice thickness $(\Delta z)$

Number of slices

Slice gap

Number of acquisitions $\left(N_{\text {acq }}\right)$

Read direction

Scan time
Supine

Spin echo

Transverse

Position for whole ankle coverage

$22 \mathrm{msec}$

$900 \mathrm{msec}$

$90^{\circ}$

$140 \mathrm{~mm}, 140 \mathrm{rm}$, with $r=5 / 7$

(rectangular field of view)

$0.27 \mathrm{~mm}, 0.39 \mathrm{~mm}$

$512,256 r$, with $r=5 / 7$ (rectangular field of view)

$4 \mathrm{~mm}$

22

$1 \mathrm{~mm}$

1

Anterior to posterior

$2 \mathrm{~min}, 56 \mathrm{sec}$

Table A25.1.5 Parameters for Transverse $T_{2}$-Weighted FSE

\begin{tabular}{ll}
\hline Patient position & Supine \\
Scan type & Fast spin echo \\
Imaging plane (orientation) & Transverse \\
Central slice or volume center & Position for whole ankle coverage \\
Echo time $\left(T_{\mathrm{E}}\right)$ & $90 \mathrm{msec}$ \\
Echo train length $(\mathrm{ETL})$ & 6 \\
Repeat time $\left(T_{\mathrm{R}}\right)$ & $3500 \mathrm{msec}$ \\
Flip angle $(\mathrm{FA})$ & $90^{\circ}$ \\
Fields of view $\left(\mathrm{FOV}_{x}, \mathrm{FOV}_{y}\right)$ & $140 \mathrm{~mm}, 140 \mathrm{rmm}$, with $r=5 / 7$ \\
& $($ rectangular field of view) \\
Resolution $(\Delta x, \Delta y)$ & $0.55 \mathrm{~mm}, 0.73 \mathrm{~mm}$ \\
Number of data points collected $\left(N_{x}, N_{y}\right)$ & $256,192 r$, with $r=5 / 7$ (rectangular \\
& field of view) \\
Slice thickness $(\Delta z)$ & $4 \mathrm{~mm}$ \\
Number of slices & 22 \\
Slice gap & $1 \mathrm{~mm}$ \\
Number of acquisitions $\left(N_{\text {acq }}\right)$ & 2 \\
Read direction & Anterior to posterior \\
Fat suppression & Yes \\
Scan time & $3 \mathrm{~min}, 3$ sec \\
\hline
\end{tabular}

The sagittal scout scan is the best plane for planning this transverse sequence. The coverage provided should then be checked on the coronal scout. This transverse sequence is used to position subsequent scans accurately.

When Achilles tendon tears are encountered, care should be taken to cover the Achilles tendon in its entirety. Often, retraction of the proximal edge of the torn tendon requires additional imaging of the calf region. In such cases, a body coil should be used to ensure complete coverage of the entire Achilles tendon and its myotendinous junction. In acute tears, imaging in plantar flexion may approximate the torn ends and can provide the surgeon with information regarding the length of the remaining tendon gap. 
Table A25.1.6 Parameters for Coronal $T_{1}$-Weighted Spin Echo

Patient position

Scan type

Imaging plane (orientation)

Central slice or volume center

Echo time $\left(T_{\mathrm{E}}\right)$

Repeat time $\left(T_{\mathrm{R}}\right)$

Flip angle (FA)

Fields of view $\left(\mathrm{FOV}_{x}, \mathrm{FOV}_{y}\right)$

Resolution $(\Delta x, \Delta y)$

Number of data points collected $\left(N_{x}, N_{y}\right)$

Slice thickness $(\Delta z)$

Number of slices

Slice gap

Number of acquisitions $\left(N_{\text {acq }}\right)$

Read direction

Scan time
Supine

Spin echo

Coronal

Position for whole ankle coverage

$22 \mathrm{msec}$

$750 \mathrm{msec}$

$90^{\circ}$

$140 \mathrm{~mm}, 140 r \mathrm{~mm}$, with $r=5 / 7$

(rectangular field of view)

$0.27 \mathrm{~mm}, 0.63 \mathrm{~mm}$

$512,224 r$, with $r=5 / 7$ (rectangular

field of view)

$4 \mathrm{~mm}$

18

$1 \mathrm{~mm}$

2

Left to right

$4 \mathrm{~min}, 5 \mathrm{sec}$

\section{Sequence 3: Transverse $T_{2}$-weighted FSE}

13. Set parameters for the $T_{2}$-weighted FSE sequence as indicated in Table A25.1.5 and run sequence 3 .

This scan is positioned as in sequence 2. The coverage should include the ankle joint and hindfoot structures as well. This sequence will provide demonstration of alterations in water content characteristic of most pathologic conditions in the foot and ankle. Fat suppressed imaging may be utilized to highlight the contrast differences between the low signal tendinous and ligamentous structures and the abnormal-free water.

Perform system shim as recommended by manufacturer as this is a fat-saturation sequence.

\section{Sequence 4: Coronal $T_{1}$-weighted spin echo}

14. Use the transverse $T_{1}$-weighted images to position the coronal scans. Set parameters for the coronal $T_{1}$-weighted spin echo as given in Table A25.1.6 and run sequence 4 .

Some angulation is needed to provide true coronal anatomic imaging. The imaging plane should be parallel to the anterior margin of the talar dome (Fig. A25.1.2). The coverage should include the entire Achilles tendon posteriorly.

This plane is particularly useful for evaluating osteochondral lesions of the talar dome, sinus tarsi, and subtalar joint abnormalities and injuries of the collateral ligaments.

\section{Sequence 5: Coronal fat-suppressed proton density-weighted fast spin echo}

15. Set parameters for the coronal fat-suppressed proton density-weighted FSE sequence as indicated in Table A25.1.7 and run sequence 5.

This scan is just positioned as sequence 4. Perform system shim as recommended by manufacturer, as this is a fat saturation sequence.

\section{Sequence 6: Sagittal turbo STIR}

16. Set parameters for the sagittal turbo STIR sequence as indicated in Table A25.1.8 and run sequence 6 .

The $T_{1}$-weighted transverse image is used to position the sagittal scans. The imaging plane should be aligned with the long axis of the calcaneus (Fig. A25.1.3). The lateral wall of the calcaneus can be used as a reference landmark to ensure appropriate angulation.

The sagittal plane is particularly useful for evaluating Achilles tendon ruptures. The entire Achilles tendon as well as its myotendinous junction should be covered.

A25.1.6 

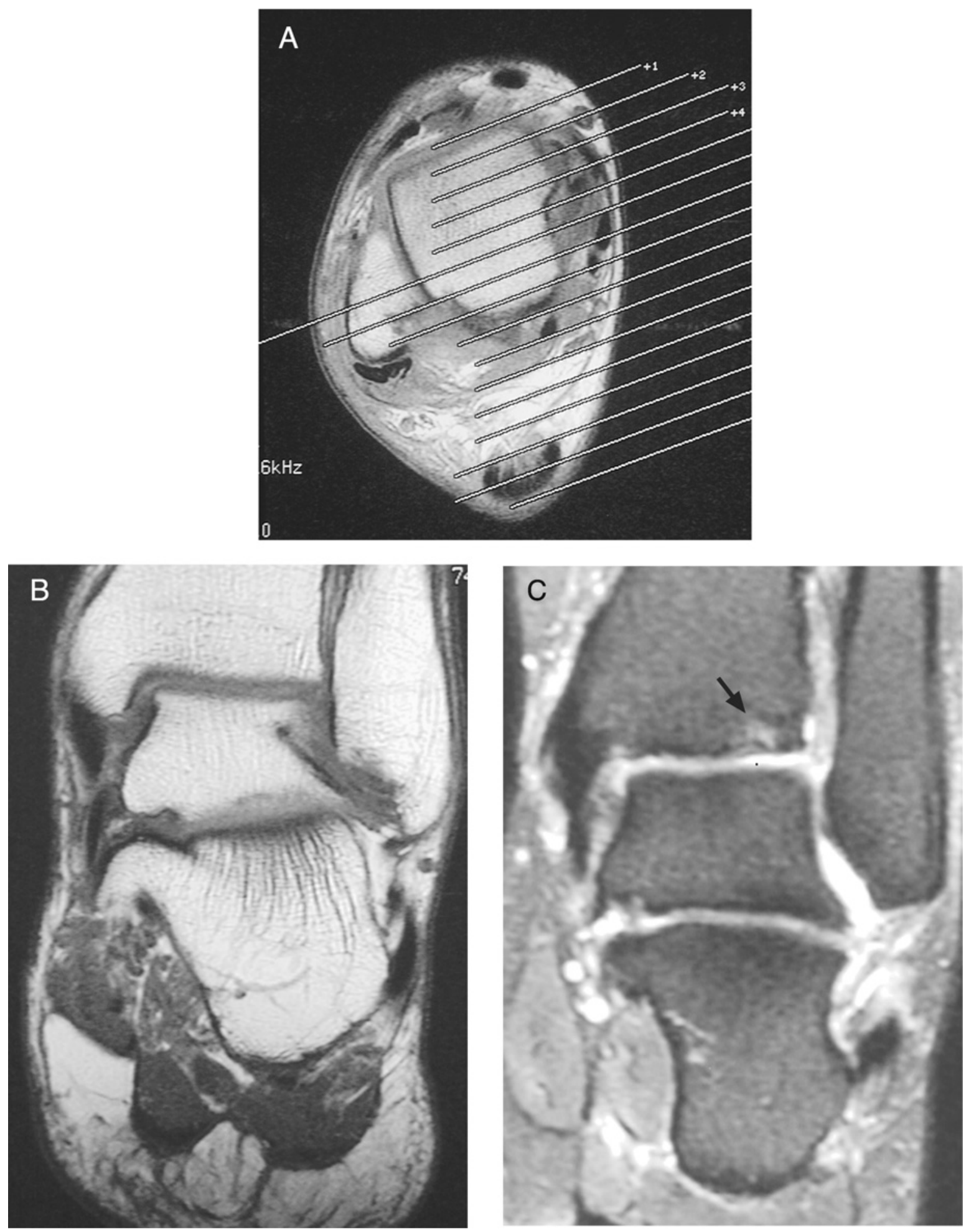

Figure A25.1.2 Coronal imaging plane. (A) Based on a transverse $T_{1}$-weighted image, the coronal plane is prescribed parallel to the anterior margin of the talar dome. Care should be taken to cover the entire Achilles posteriorly. (B) Coronal $T_{1}$-weighted image through the posterior aspect of the talus. (C) Coronal fat suppressed proton density weighted FSE image in a different patient. Note a small osteochondral lesion of the tibia (short arrow). 
Table A25.1.7 Parameters for Coronal Fat Suppressed Proton Density Weighted Fast Spin Echo

Patient position

Scan type

Imaging plane (orientation)

Central slice or volume center

Echo time $\left(T_{\mathrm{E}}\right)$

Echo train length (ETL)

Repeat time $\left(T_{\mathrm{R}}\right)$

Flip angle (FA)

Fields of view $\left(\mathrm{FOV}_{x}, \mathrm{FOV}_{y}\right)$

Resolution $(\Delta x, \Delta y)$

Number of data points collected $\left(N_{x}, N_{y}\right)$

Slice thickness $(\Delta z)$

Number of slices

Slice gap

Number of acquisitions ( $\left.N_{\text {acq }}\right)$

Read direction

Fat suppression

Scan time

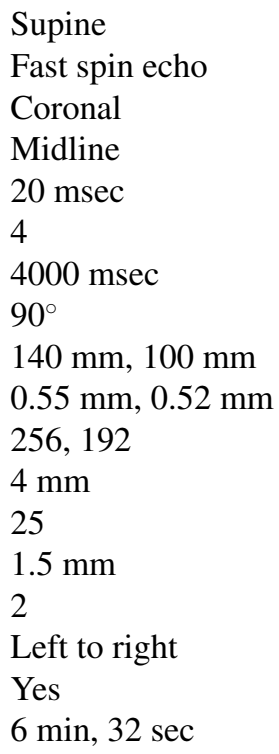

Supine

Fast spin echo

Coronal

Midline

$20 \mathrm{msec}$

4

$4000 \mathrm{msec}$

$90^{\circ}$

$140 \mathrm{~mm}, 100 \mathrm{~mm}$

$0.55 \mathrm{~mm}, 0.52 \mathrm{~mm}$

256, 192

$4 \mathrm{~mm}$

25

$1.5 \mathrm{~mm}$

2

Left to right

Yes

$6 \mathrm{~min}, 32 \mathrm{sec}$

Table A25.1.8 Parameters for Sagittal Turbo STIR

\begin{tabular}{ll} 
Patient position & Supine \\
Scan type & STIR fast spin echo \\
Imaging plane (orientation) & Sagittal \\
Central slice or volume center & Midline \\
Echo time $\left(T_{\mathrm{E}}\right)$ & $21 \mathrm{msec}$ \\
Echo train length $(\mathrm{ETL})$ & 6 \\
Repeat time $\left(T_{\mathrm{R}}\right)$ & $4200 \mathrm{msec}$ \\
Inversion time $\left(T_{\mathrm{I}}\right)$ & $130 \mathrm{msec}$ \\
Flip angle $(\mathrm{FA})$ & $90^{\circ}$ \\
Fields of view $\left(\mathrm{FOV}, \mathrm{FOV}_{y}\right)$ & $150 \mathrm{~mm}, 150 \mathrm{~mm}$ \\
Resolution $(\Delta x, \Delta y)$ & $0.59 \mathrm{~mm}, 0.78 \mathrm{~mm}$ \\
Number of data points collected $\left(N_{x}, N_{y}\right)$ & 256,192 \\
Slice thickness $(\Delta z)$ & $4 \mathrm{~mm}$ \\
Number of slices & 14 \\
Slice gap & $1 \mathrm{~mm}$ \\
Number of acquisitions $\left(N_{\text {acq }}\right)$ & 2 \\
Read direction & Cranial to caudal \\
Scan time & $4 \mathrm{~min}, 33 \mathrm{sec}$ \\
\hline
\end{tabular}

\section{Sequence 7: Post-gadolinium fat-suppressed $T_{1}$-weighted spin echo (optional)}

When osteomyelitis or neoplastic processes are suspected, additional imaging following i.v. administration of gadolinium is recommended.

17. Pull the patient out of the magnet.

18. Establish an i.v. line from which the contrast agent can be injected, and attach this line securely to the patient so that movement into or out of the magnet will not pull at the patient's arm.

It is preferable to insert the line prior to imaging and to leave the patient in the magnet so that there is no intervening motion between the scans run before contrast agent injection and those run after injection. 

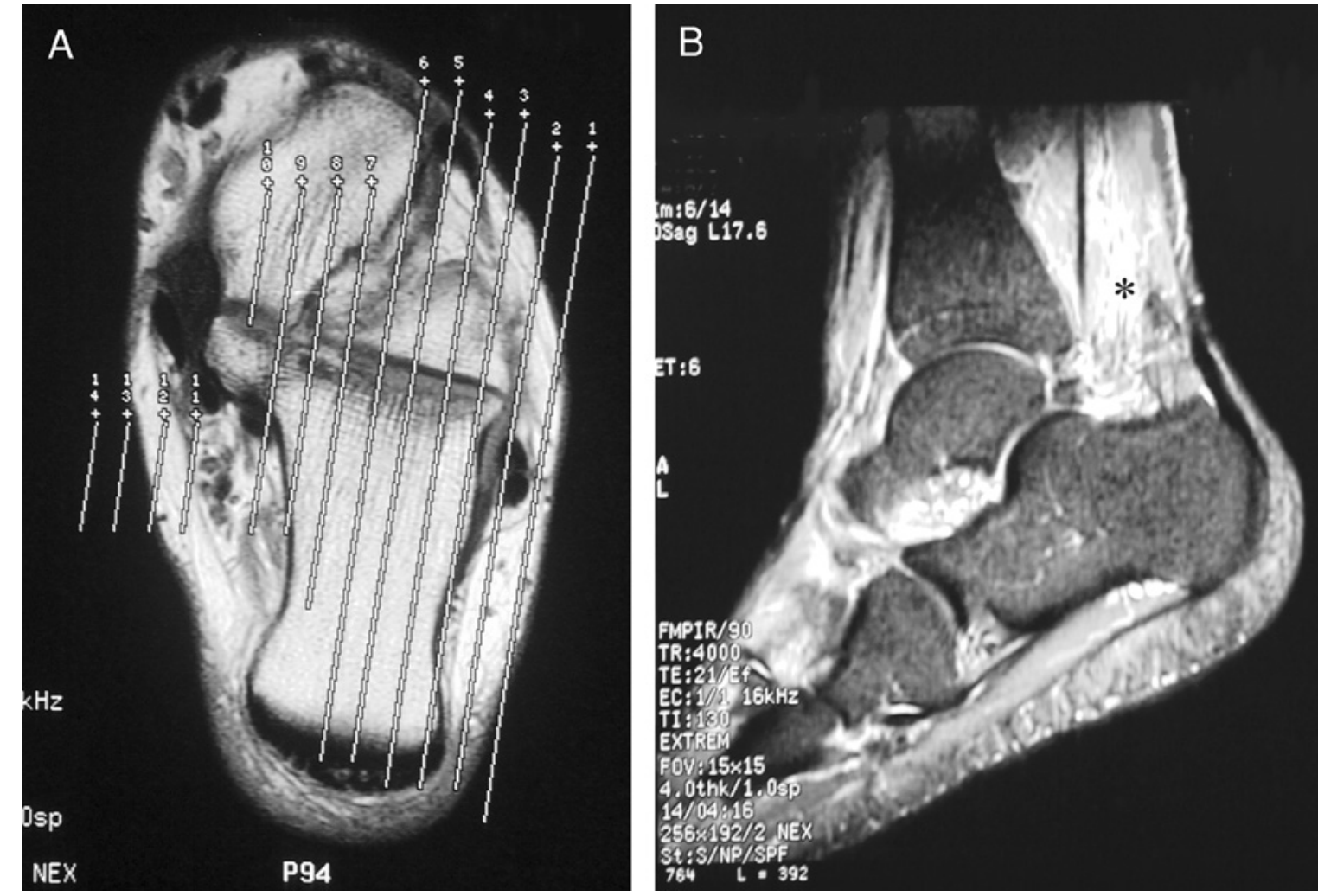

Figure A25.1.3 Sagittal imaging plane. (A) Based on a transverse $T_{1}$-weighted image, the sagittal plane should be aligned with the long axis of the calcaneus. The lateral wall of the calcaneus is used as a reference landmark to ensure appropriate angulation. (B) Sagittal STIR image. Edematous changes of the pre-Achilles fat pad are noted (asterisk) in this patient with diagnosis of anterior Achilles peritendinitis.

Table A25.1.9 Parameters for Post-Gadolinium Fat-Suppressed $T_{1}$-Weighted Spin Echo

Patient position

Scan type

Imaging plane (orientation)

Central slice or volume center

Echo time $\left(T_{\mathrm{E}}\right)$

Repeat time $\left(T_{\mathrm{R}}\right)$

Flip angle (FA)

Fields of view $\left(\mathrm{FOV}_{x}, \mathrm{FOV}_{y}\right)$

Resolution $(\Delta x, \Delta y)$

Number of data points collected $\left(N_{x}, N_{y}\right)$

Slice thickness $(\Delta z)$

Number of slices

Slice gap

Number of acquisitions $\left(N_{\text {acq }}\right)$

Read direction

Fat suppression

Scan time
Supine

Spin echo

Transverse and coronal

Position for whole-ankle coverage

Minimum

$600 \mathrm{msec}$

$90^{\circ}$

$140 \mathrm{~mm}, 100 \mathrm{~mm}$

$0.55 \mathrm{~mm}, 0.52 \mathrm{~mm}$

256, 192

$3 \mathrm{~mm}$

22

$1 \mathrm{~mm}$

2

Anterior to posterior (for transverse) left to right (for coronal)

Yes

$3 \mathrm{~min}, 54 \mathrm{sec}$ 
19. Repeat step 9.

20. If the patient moved, run sequence 1 again to ensure the position of the patient.

21. Leaving the patient in the magnet, inject the contrast agent, and flush the line with $10 \mathrm{ml}$ saline.

A dose of $0.1 \mathrm{mmol} / \mathrm{kg}$ of contrast agent is usually given.

22. Run sequence 7 according to Table A25.1.9 with a transverse orientation first and then coronal.

\section{COMMENTARY}

\section{Background Information}

MR imaging, based on its unique soft-tissue contrast resolution, noninvasiveness, and multiplanar capabilities, has been applied to the assessment of tendinous, ligamentous, and osseous abnormalities of the foot and ankle (Erickson and Johnson, 1997). In general, $T_{1}$-weighted images provide high anatomical detail while $T_{2}$-weighted images are useful to evaluate alterations in water content characteristic of most pathologic conditions. Short tau inversion recovery (STIR) and fatsuppressed imaging may be utilized to highlight the contrast differences between the low signal tendinous and ligamentous structures and the abnormal-free water. These sequences are also very useful for assessment of bone marrow edema.

MR imaging of the foot and ankle should be obtained in the transverse, sagittal, and coronal planes. The transverse and sagittal planes provide most of the information necessary to assess the tendons at the ankle. The transverse images are optimal for evaluating tendon morphology, longitudinal splits, tendon sheath fluid, and adjacent soft tissues such as overlying retinacula. The sagittal images are most useful for depicting disease of the Achilles tendon. Oblique transverse imaging, perpendicular to the long axis of the tendons, has been recommended but the authors find it unnecessary. Kinematic MR imaging in the transverse plane has been proposed as an ancillary method for dynamic visualization of the peroneal tendons during dorsiflexed and plantar-flexed ankle movements (Shellock et al., 1997). This technique allows identification of chronic occult subluxation and dislocation. Since repetitive attritional trauma of the peroneal tendons in and out of the fibular groove has been associated with subsequent development of partial longitudinal tears of these structures, early diagnosis by kinematic MR imaging may help to prevent this complication. High-resolution kinematic MR images can be obtained using a device incorporating dual 5 -in. circular surface coils. Transverse short $T_{\mathrm{R}}$ gradient echo (GRASS: gradient refocused acquisition in the steady state) images with $T_{\mathrm{R}} / T_{\mathrm{E}}$ of $6.1 \mathrm{msec} /$ $2.5 \mathrm{msec}$ and 5-mm slice thickness have been used.

The ligaments of the ankle joint are best evaluated on transverse and coronal images. Early MR studies advocated imaging with the foot in both dorsiflexion and plantar flexion to optimally visualize each ligament in its entirety on a single cut. Conversely, recent evidence based on correlation of MR imaging and anatomic findings, suggest that coronal and transverse slices with the ankle taped in $10^{\circ}$ to $20^{\circ}$ of dorsiflexion are sufficient to provide detailed evaluation of the anatomy of the lateral and medial collateral ligaments (Muhle et al., 1999). In the authors' experience, however, routine examinations with the ankle in neutral position are usually sufficient for diagnosing most ligamentous abnormalities of the ankle.

Routine MR imaging is adequate for visualizing most osseous lesions of the hindfoot. However, the use of two 3-in. planar coils placed adjacent to the medial and lateral malleoli has been recommended for high resolution imaging of the talar dome in patients with suspected osteochondral lesions (Verhaven et al., 1991). The field of view can be reduced to $8 \mathrm{~cm}$ using acquisition matrices of 256 by 192 to 256 by 256 . Coronal fat suppressed $T_{2}$-weighted fast spin echo (Table A25.1.10) or $T_{2}{ }^{*}$ gradient echo sequences (Table A25.1.11) are best for evaluating the subchondral bone and status of the articular cartilage.

MR imaging diagnosis of fragment stability has relied on $T_{2}$-weighted images. A low signal intensity line in the interface between the normal bone and the osteochondral fragment has been related to healing and stability as opposed to a high signal intensity interface, which indicates fluid in between the nonattached part of the fragment and the donor site 
Table A25.1.10 Coronal $T_{2}$-Weighted Fast Spin Echo Sequence

$\begin{array}{ll}\text { Patient position } & \text { Supine } \\ \text { Scan type } & \text { Fast spin echo } \\ \text { Imaging plane (orientation) } & \text { Coronal } \\ \text { Central slice or volume center } & \text { Midline } \\ \text { Echo time }\left(T_{\mathrm{E}}\right) & 45 \mathrm{msec} \\ \text { Echo train length }(\mathrm{ETL}) & 4 \\ \text { Repeat time }\left(T_{\mathrm{R}}\right) & 4000 \mathrm{msec} \\ \text { Flip angle }(\mathrm{FA}) & 90^{\circ} \\ \text { Fields of view }\left(\mathrm{FOV}_{x}, \mathrm{FOV}_{y}\right) & 100 \mathrm{~mm}, 100 \mathrm{~mm} \\ \text { Resolution }(\Delta x, \Delta y) & 0.39 \mathrm{~mm}, 0.52 \mathrm{~mm} \\ \text { Number of data points collected }\left(N_{x}, N_{y}\right) & 256,192 \\ \text { Slice thickness }(\Delta z) & 4 \mathrm{~mm} \\ \text { Number of slices } & 25 \\ \text { Slice gap } & 1.5 \mathrm{~mm} \\ \text { Number of acquisitions }\left(N_{\text {acq }}\right) & 2 \\ \text { Read direction } & \text { Left to right } \\ \text { Fat suppression } & \text { Yes } \\ \text { Scan time } & 6 \mathrm{~min}, 32 \mathrm{sec}\end{array}$

Table A25.1.11 Coronal $T_{2}{ }^{*}$-Gradient Echo Sequence

\begin{tabular}{ll}
\hline Patient position & Supine \\
Scan type & 2-D gradient echo \\
Imaging plane (orientation) & Coronal \\
Central slice or volume center & Midline \\
Echo time $\left(T_{\mathrm{E}}\right)$ & $15 \mathrm{msec}$ \\
Repeat time $\left(T_{\mathrm{R}}\right)$ & $400 \mathrm{msec}$ \\
Flip angle $(\mathrm{FA})$ & $15^{\circ}$ \\
Fields of view $\left(\mathrm{FOV}_{x}, \mathrm{FOV}_{y}\right)$ & $100 \mathrm{~mm}, 100 \mathrm{~mm}$ \\
Resolution $(\Delta x, \Delta y)$ & $0.20 \mathrm{~mm}, 0.45 \mathrm{~mm}$ \\
Number of data points collected $\left(N_{x}, N_{y}\right)$ & 512,224 \\
Slice thickness $(\Delta z)$ & $3 \mathrm{~mm}$ \\
Number of slices & 25 \\
Slice gap & $3 \mathrm{~mm}$ (interleave) \\
Number of acquisitions $\left(N_{\text {acq }}\right)$ & 2 \\
Read direction & Left to right \\
Fat suppression & No \\
Scan time & $6 \mathrm{~min}, 5 \mathrm{sec}$ \\
\hline
\end{tabular}

(Mesgarzadeh et al., 1987). However, the inability to discriminate between fluid and granulation tissue on $T_{2}$-weighted images, has led some investigators to utilize MR arthrography in this setting (Chandnani, 1994). Direct MR arthrography and post intraarticular contrast injection (Vahlensieck et al., 1996; Allman et al., 1999), as well as indirect MR arthrography, post-intravenous contrast injection, have also been recommended for assessing abnormalities of the articular cartilage.

Because osteochondral lesions may be complicated by osteonecrosis, assessment of fragment viability is also of significance in the MR imaging analysis. Additional evaluation may be obtained using post-intravenous gadolinium fat-suppressed $T_{1}$-weighted images, which demonstrate marrow enhancement in viable bone and lack of enhancement in necrotic fragments.

Depiction of bone marrow signal abnormality manifested as low signal intensity on $T_{1}$-weighted images and $T_{2}$-weighted hyperintensity images in continuity with a skin ulcer or adjacent subcutaneous abscess are indicative of osteomyelitis. Distinguishing features with neuroarthropathy include bone marrow changes in the absence of adjacent soft tissue infection. Gadolinium-enhanced MR imaging using fat-suppressed $T_{1}$-weighted images in the sagittal and short axis planes may provide additional information regarding the extent of
Ankle and Foot

A25.1.11 
soft tissue abnormality as well as depiction of sinus tracts and abscesses (Morrison et al., 1998).

\section{Critical Parameters and Troubleshooting}

Motion artifacts can be reduced by carefully placing and securing the ankle joint within the extremity coil. Wedging sponges should be used in order to provide stability. If these measures fail, conscious sedation can be administered. If sedation is not possible, shortening the protocol can be attempted by reducing the acquisition matrix. This will, however, decrease resolution.

Phase encoding artifact may arise from the posterior neurovascular bundle. On transverse images, the flow related artifact could obscure tendinous or ligamentous structures. In such cases, changing the phase encoding direction from anterior/posterior to right/left usually solves this problem.

\section{Anticipated Results}

This protocol is designed to assess ligamentous, tendinous, and osseous abnormalities that affect the ankle joint. The use of post gadolinium imaging is indicated when osteomyelitis and neoplastic processes are suspected.

\section{Literature Cited}

Allman, K.H., Schafer, O., Haver, M., Winterer, J., Laubenberger, J., Reichelt, A., and Uhl, M. 1999. Indirect MR arthrography of the unexercised glenohumeral joint in patients with rotator cuff tears. Invest. Radiol. 34:435-440.

Chandnani, V.P., Harper, M.T., Ficke, J.R., Gagliardi, J.A., Rolling, L., Christensen, K.P., and Hansen, M.F. 1994. Chronic ankle instability: Evaluation with MR arthrography, MR imaging and stress radiography. Radiology 192:189-194.

Erickson, S.J. and Johnson, J.E. 1997. MR imaging of the ankle and foot. Radiol. Clin. N. Am. 35:163-192.
Kneeland, J.B. 1994. Technical considerations for magnetic resonance imaging of the ankle and foot. Magn. Reson. Imaging Clin. N. Am. 2:2328.

Mesgarzadeh, M., Sapega, A.A., Bonakdarpour, A., Revesz, G., Moyer, R.A., Maurer, A.H., and Alburger, P.D. 1987. Osteochondritis dissecans: Analysis of mechanical stability with radiography, scintigraphy, and MR imaging. Radiology 165:775-780.

Morrison, W.B., Schweitzer, M.E., Batte, W.G., Radack, D.P., and Russel, K.M. 1998. Osteomyelitis of the foot: Relative importance of primary and secondary MR imaging signs. Radiology 201:625-632.

Muhle, C., Frank, L.R., Rand, T., Yeh, L., Wong, E.C., Skaf, A., Dantas, R.W., Haghighi, P., Trudell, D., and Resnick, D. 1999. Collateral ligament of the ankle: High resolution MR imaging with a local gradient coil and anatomic correlation in cadavers. Radiographics 19:673-683.

Shellock, F.G. and Kanal, E. 1996. Magnetic Resonance: Bioeffects, Safety, and Patient Management, 2nd ed. Lippincott-Raven, Philadelphia.

Shellock, F.G., Feske, W., and Frey, C. 1997. Peroneal tendons: Use of kinematic MR imaging of the ankle to determine subluxation. J. Magn. Reson. Imaging 7:451-454.

Vahlensieck, M., Peterfy, C.G., Wischer, T., Sommer, T., Lang, P., Schlippert, U., Genant, H.K., and Schild, H.H. 1996. Indirect MR arthrography: Optimization and clinical applications. Radiology 200:249-254.

Verhaven, E.F., Shahabpour, M., Handelberg, F.W.J., Vaes, P.H., and Opdecam, P.J. 1991. The accuracy of three-dimensional magnetic resonance imaging in the diagnosis of ruptures of the lateral ligaments of the ankle. Am. J. Sports Med. 19:583-587.

Zlatkin, M.B. 1999. Techniques for MR imaging of joints in sports medicine. Magn. Reson. Imaging Clin. N. Am. 7:1-21.

Contributed by Jenny Bencardino and Zehava S. Rosenberg

Hospital for Joint Diseases

NYU Medical Center

New York, New York
MR Imaging of the Ankle and Hindfoot

A25.1.12 